\title{
Prolonged non-survival in PICU: does a do-not-attempt-resuscitation order matter
}

\author{
Kam Lun E Hon ${ }^{1 *}$, Terence Chuen Wai Poon ${ }^{1}$, William Wong ${ }^{1}$, Kin Kit Law², Hiu Wing Mok ${ }^{2}$, Ka Wing Tam², \\ Wai Kin Wong ${ }^{2}$, Hiu Fung $\mathrm{Wu}^{2}$, Ka Fai To ${ }^{3}$, Kam Lau Cheung ${ }^{1}$, Hon Ming Cheung', Ting Fan Leung ${ }^{1}$, \\ Chi Kong Li ${ }^{1}$ and Alexander KC Leung ${ }^{4}$
}

\begin{abstract}
Background: Etiologies of pediatric intensive care unit (PICU) mortality are diverse. This study aimed to investigate the pattern of PICU mortality in a regional trauma center, and explore factors associated with prolonged non-survival.

Methods: Demographic data of all PICU deaths in a regional trauma center were analyzed. Factors associated with prolonged nonsurvival (length of stay) were investigated with univariate log rank and multivariate Cox-Regression forward stepwise tests.

Results: There were 88 deaths (males 61\%; infants 23\%) over 10 years (median PICU stay $=3.5$ days, interquartile range: 1 and 11 days). The mean annual mortality rate of PICU admissions was 5.8\%. Septicemia with gram positive, gram negative and fungal pathogens were present in $13(16 \%), 13(16 \%)$ and $4(5 \%)$ of these patients, respectively. Viruses were isolated in 25 patients (28\%). Ninety percent of these 88 patients were ventilated, $75 \%$ required inotropes, $92 \%$ received broad spectrum antibiotic coverage, $32 \%$ received systemic corticosteroids, $56 \%$ required blood transfusion and 39\% received anticonvulsants. Thirty nine patients (44\%) had a DNAR (Do-Not-Attempt-Resuscitation) order with their deaths at the PICU. Comparing with non-trauma category, trauma patients had higher mortality score, no premorbid disease, suffered asystole preceding PICU admission and subsequent brain death. Oncologic conditions were the most prevalent diagnosis in the non-trauma category. There was no gunshot or asthma death in this series. Prolonged non-survival was significantly associated with DNAR, fungal infections, and mechanical ventilation but negatively associated with bacteremia.
\end{abstract}

Conclusions: Death in the PICU is a heterogeneous event that involves infants and children. Resuscitation was not attempted at the time of their deaths in nearly half of the patients in honor of parents' wishes. Parents often make DNAR decision when medical futility becomes evident. They could be reassured that DNAR did not mean "abandoning" care. Instead, DNAR patients had prolonged PICU stay and received the same level of PICU supports as patients who did not respond to cardiopulmonary resuscitation.

Keywords: Bacteria, Fungus, PICU, Pediatric intensive care, Malignancy, Mortality, Oncology, PIM2, Sepsis, Trauma, Virus, Do-not-attempt-resuscitation (DNAR), Not-responding-to-cardiopulmonary-resuscitation (NRCPR), Brain death, Organ donation

\footnotetext{
* Correspondence: ehon@hotmail.com

${ }^{1}$ Department of Pediatrics, The Chinese University of Hong Kong, 6/F, Clinical

Science Building, Prince of Wales Hospital, Shatin, Hong Kong, SAR, China

Full list of author information is available at the end of the article
} 


\section{Background}

Improvements in resuscitation, access to advanced technologies and access to newer medications have led to improved survival of critically ill patients in intensive care units [1-4]. Nevertheless, children are occasionally inflicted with severe and life threatening diseases, necessitating supports and treatment at a pediatric intensive care unit (PICU) [5-7]. Unfortunately, a small percentage would not survive despite optimal PICU care and support [4,8].

Hong Kong, with a population of over 7 million people, has a dual public and private system for both primary and secondary health care. Although there are 10 government (Hospital Authority [HA]) and 10 private hospitals providing general pediatric inpatient service, the HA system provides the majority of inpatient pediatric care. The Prince of Wales Hospital (PWH) is a university teaching/government hospital and a tertiary trauma center situated in the New Territories in Hong Kong. PWH provides tertiary pediatric intensive care unit (PICU) service for children $<12$ years of age to this region with a catchment population of over 1.1 million (approximately 25\% were children $<12$ years of age). The incidence and pattern of mortality in young children reflects the social and demographic characteristics of that city [2,8-10]. It is generally observed that severe infections significantly contribute to childhood mortality in developing nations, whereas malignancy may be a key cause of mortality in industrialized or commercialized nations [8]. PICU mortality is diverse and broadly divided into two mutually exclusive categories: trauma and non-trauma etiologies $[11,12]$. This study aimed to investigate the pattern of mortality in young children admitted to a PICU in a regional trauma center, and explore factors associated with prolonged non-survival.

\section{Methods}

The criteria for PICU admissions include cardiopulmonary insufficiency/failure, neurological deterioration, and concerns of emergency care and/or general pediatric staff. Cases were identified from the hospital's computerized auditing system and from the PICU database of every admission to the unit. The PICU database was audited monthly. Every child who died in PICU from October 2002 (PICU opening) to December 2012 was recruited. Data were collected from patient records, hospital Clinical Management System (CMS) and autopsy reports, which included age, gender, duration in PICU stay, primary diagnosis, cause of death, mode of death, underlying conditions; use of inotropes, systemic antibiotics, anticonvulsants and systemic corticosteroids; ventilatory support; and blood product transfusions. The data also included patients' clinical conditions such as asystole preceding PICU admission, diagnosis of encephalitis or raised intracranial pressure (ICP), disseminated intravascular coagulopathy (DIC) and multi-organ system failure (MOSF). The Paediatric index of mortality (PIM2) score was calculated for each patient (http://www.sfar.org/scores2/pim22. html) [13]. According to PIM2, high risk diagnoses include cardiac arrest preceding ICU admission, severe combined immune deficiency, leukemia or lymphoma after first induction, spontaneous cerebral hemorrhage, cardiomyopathy or myocarditis, hypoplastic left heart syndrome, HIV infection, liver failure, and neuro-degenerative disorder. Low risk diagnoses include asthma, bronchiolitis, croup, obstructive sleep apnea and diabetic ketoacidosis. Primary diagnoses were divided into 3 categories: oncologic, injury and miscellaneous.

The respiratory viruses were identified using conventional diagnostic methods, including direct immunofluorescence testing (DIFT) on respiratory samples (e.g. nasopharyngeal aspirates [NPAs]; broncho-alveolar lavages [BALs], tracheal aspirates [TAs], and oral swabs), with confirmation and typing by type-specific DIFT and viral culture. The results of all other bacterial and fungal cultures on respiratory specimens (including tracheal aspirate, pleural and sputum specimens), blood, cerebrospinal and deep wound cultures were recorded. Mode of death was classified into two categories: "Do Not Attempt Resuscitation (DNAR)" and "Not Responding to Cardiopulmonary Resuscitation (NRCPR)". There were 5 data abstractors and inter-rater variability was controlled by standard procedures. The Ethics Committee of the Chinese University of Hong Kong approved this anonymous review with no patient identity in the manuscript, and that patient consent was not necessary for this retrospective survey. Numerical data were compared with Mann-Whitney test and categorical data with Fisher exact test. All comparisons were made twotailed, and $p$-values less than 0.05 considered statistically significant.

\section{Results}

From October 2002 to December 2012, 88 deaths occurred in 1505 admissions to the PICU (Table 1: males 61\%; infants 23\%; median PICU stay $=3.5$ days, IQR: 1 and 11 days). The mean annual mortality rate of PICU admissions was $5.8 \%$ (Table 2). There was no significant change in annual mortality over the past 10 years. The median PIM2 score was $20 \%$. Ninety percent of these patients were ventilated, $75 \%$ required inotropes, $92 \%$ received broad spectrum antibiotic coverage, $32 \%$ received systemic corticosteroids, 56\% blood transfusion and 39\% anticonvulsants. Thirty nine patients $(44 \%)$ had a DNAR (Do-Not-Attempt-Resuscitation order) prior to their death at the PICU, but few were actively withdrawn.

Twenty four were oncological diagnoses, 9 were trauma/ injuries and 55 miscellaneous diagnoses which included various infections, one case each of acute disseminated encephalomyelitis, status epilepticus, dilated cardiomyopathy, 
Table 1 Clinical data of patients who died in the PICU

\begin{tabular}{|c|c|c|c|}
\hline Characteristic & Trauma $(n=9)$ & Non-trauma $(n=79)$ & $P$ \\
\hline Median age, year (interquartile range) & $5.6(2.4,10.7)$ & $4.0(0.9,7.4)$ & 0.20 \\
\hline Male, n (\%) & $8(88 \%)$ & $46(58 \%)$ & 0.15 \\
\hline PIM2 score\%, median (interquartile range) & $90(60,92)$ & $12(5,56)$ & 0.002 \\
\hline Oncological diagnosis, n (\%) & $0(0 \%)$ & $24(30 \%)$ & 0.11 \\
\hline \multicolumn{4}{|l|}{ Pathogens in blood culture, n (\%): } \\
\hline Gram + bacteria & $0(0 \%)$ & $13(16 \%)$ & 0.35 \\
\hline Gram - bacteria & $0(0 \%)$ & $13(16 \%)$ & 0.35 \\
\hline Fungal & $0(0 \%)$ & $4(5 \%)$ & 1.00 \\
\hline Virus isolation, n (\%) & $0(0 \%)$ & $25(32 \%)$ & 0.055 \\
\hline Mechanical ventilation, n (\%) & $9(100 \%)$ & $71(90 \%)$ & 1.00 \\
\hline Inotropes, n (\%) & $8(89 \%)$ & $59(75 \%)$ & 0.68 \\
\hline Anticonvulsants, n (\%) & $3(33 \%)$ & $32(41 \%)$ & 1.00 \\
\hline Blood transfusion, n (\%) & $4(44 \%)$ & $45(57 \%)$ & 0.50 \\
\hline Platelets or fresh frozen plasma, n (\%) & $5(56 \%)$ & $49(62 \%)$ & 0.73 \\
\hline Disseminated intravascular coagulopathy, n (\%) & $3(33 \%)$ & $37(47 \%)$ & 0.50 \\
\hline Systemic antibiotics, n (\%) & $6(67 \%)$ & 75 (95\%) & 0.022 \\
\hline Systemic corticosteroids, n (\%) & $0(0 \%)$ & $28(35 \%)$ & 0.052 \\
\hline PICU survival, median (interquartile range) & $2.0(1.0,6.0)$ & $3.0(1.0,11.0)$ & 0.84 \\
\hline Repeated PICU admission, n (\%) & $0(0 \%)$ & 19 (24\%) & 0.20 \\
\hline DNAR, n (\%) & $5(56 \%)$ & $34(43 \%)$ & 0.50 \\
\hline NRCPR, n (\%) & $4(44 \%)$ & $45(57 \%)$ & \\
\hline Brain death confirmed, $\mathrm{n}(\%)$ & $4(44 \%)$ & $7(9 \%)$ & 0.011 \\
\hline Asystole before PICU, n (\%) & $5(56 \%)$ & $10(13 \%)$ & 0.006 \\
\hline Post-mortem examination, n (\%) & $3(33 \%)$ & $22(28 \%)$ & 0.71 \\
\hline
\end{tabular}

Table 2 PICU deaths as a percentage of the annual PICU admissions

\begin{tabular}{ccc}
\hline PICU admissions & Total & Mortality \\
\hline From Oct 2002 & 33 & $3(9.1)$ \\
2003 & 122 & $10(8.2)$ \\
2004 & 155 & $7(4.5)$ \\
2005 & 112 & $4(3.6)$ \\
2006 & 127 & $8(6.3)$ \\
2007 & 144 & $11(7.6)$ \\
2008 & 138 & $11(8.0)$ \\
2009 & 140 & $4(2.9)$ \\
2010 & 149 & $6(4.0)$ \\
2011 & 198 & $12(5.6)$ \\
2012 & 187 & $12(8.3)$ \\
Total & $\mathbf{1 5 0 5}$ & $\mathbf{8 8}(5.8)$ \\
\hline$p$ for trend & & $>0.5$ \\
\hline
\end{tabular}

hypertrophic cardiomyopathy, liver failure, post-operative bleeding, mucopolysaccharidosis type I, partial pyruvate dehydrogenase deficiency, tetrahydrobiopterin deficiency with toxic epidermolysis, spinal muscular atrophy with aspiration pneumonia, Down syndrome, multiple congenital anomalies and respiratory failure, and two cases of undetermined etiology. There was no PIM2 low-risk-diagnosis in this series (http://www.sfar.org/scores2/pim22.html) [13].

\section{Infections}

Eighty one (92\%) of patients had been treated with antibiotics for clinical infection. Septicemia with gram positive, gram negative and fungal pathogens were present in $13(16 \%), 13(16 \%)$ and $4(5 \%)$ of these patients, respectively. The most common gram negative pathogens isolated in the blood cultures were pseudomonas aeruginosa $(\mathrm{n}=5)$, E. coli $(\mathrm{n}=3)$, enterobacter $(\mathrm{n}=2)$, stenotrophomonas $(\mathrm{n}=2)$ and chryseobacterium $(\mathrm{n}=2)$. The most common gram positive pathogens isolated in blood was coagulasenegative staphylococcus species (CNSS) $(\mathrm{n}=5)$. There were two case of pneumococcal deaths associated with multiorgan system failure and hemolytic uremic syndrome. 
Among the fungal blood cultures were candida parasilosis, candida albicans, candida tropicalis, penicillium marneffei; 2 also had concurrent bacterial septicemia.

Viruses were isolated in 25 patients (28\%); 7 of these patients also had concurrent bacterial septicemia and 10 patients with underlying hematologic/oncologic diagnoses. The viruses included cytomegalovirus $(n=8)$, Ebstein-Barr virus $(\mathrm{n}=4)$, herpes simplex type $1(\mathrm{n}=3)$, human herpesvirus type $6(n=1)$, human herpesvirus type $7(n=1)$, respiratory syncytial virus $(n=3)$, influenza $A(n=1)$, parainfluenza type $3(\mathrm{n}=1)$, adenovirus $(\mathrm{n}=3)$, norovirus $(n=1)$, coxsackie virus $(n=1)$ and human metapneumovirus $(\mathrm{n}=1)$. There were no deaths associated with meningitis.

\section{Oncology}

Hematological and miscellaneous malignancies were a prevalent category associated with PICU deaths (including acute lymphoblastic leukemia, acute myeloblastic leukemia, chronic myeloblastic, lymphoma, hemophagocytic lymphohistiocytosis, Langerhans cell histiocytosis, medulloblastoma, glioblastoma, hepatoblastoma, neuroblastoma and mixed germ cell tumor. Septicemia was associated with hematological/malignant conditions. The majority of these patients did not present immediately post-bone marrow transplantation.

\section{Trauma}

Nine deaths $(10 \%)$ were due to trauma/injuries. Causes include traumatic head injury from fall $(\mathrm{n}=4)$, strangulation $(\mathrm{n}=1)$, suspected non accidental injury and cerebral hemorrhage $(\mathrm{n}=1)$, submersion injury $(\mathrm{n}=2)$ and suffocation $(n=1)$. There was no gunshot death or road traffic injury. Comparing with non-trauma diagnoses, trauma patients had higher PIM2 scores $(\mathrm{p}=0.002)$, were more likely to present with cardiac arrest preceding ICU admission, and to have brain death subsequently (Table 1 ). Trauma deaths typically involved healthy boys and not involving any pathogens. There were only 2 cases of organ donation following brain deaths and both were from non-Chinese families (US and Japanese).

\section{Factors associated with length of PICU stay}

Univariate analysis by log rank test and Kaplan-Meier plot and multivariate Cox-Regression forward stepwise test showed prolonged non-survival (length of PICU stay) was significantly associated with DNAR [18.6 versus 7.5 days, $\mathrm{p}<0.001$ ], fungal infections [29.6 versus 8.3 days, $\mathrm{p}=0.002$ ], and mechanical ventilation [13.7 versus 1 day, $\mathrm{p}<0.001$ ], but negatively associated with bacteremia [4.6 versus 15.5 days, $\mathrm{p}=0.031]$ (Tables 3, 4 and 5).
Table 3 Length-of-survival analysis by univariate log rank (Mantel-Cox) test

\begin{tabular}{lccc}
\hline & $\begin{array}{c}\text { Mean (standard error) } \\
\text { days }\end{array}$ & $\begin{array}{c}\text { 95\% confidence } \\
\text { interval }\end{array}$ & $\boldsymbol{P}$ \\
\hline DNAR & $18.6(5.2)$ & $8.4-28.8$ & $<0.001$ \\
NRCPR & $7.5(3.1)$ & $1.4-13.6$ & \\
No fungal infection & $8.3(2.3)$ & $3.8-12.9$ & 0.002 \\
Fungal isolates & $29.6(10.8)$ & $8.4-50.8$ & \\
No bacteremia & $15.5(4.0)$ & $7.7-23.3$ & 0.031 \\
Bacteremia & $4.6(1.4)$ & $2.0-7.3$ & \\
No antibiotics & $1.7(1.1)$ & $0.0-3.8$ & 0.008 \\
Systemic antibiotics & $13.4(3.2)$ & $7.2-19.7$ & \\
No ventilation & $1.0(0.4)$ & $0.2-1.8$ & $<0.001$ \\
Mechanical & $13.7(3.2)$ & $7.4(19.9)$ & \\
ventilation & & & \\
\hline
\end{tabular}

Length-of-survival was not associated with gender, oncological diagnosis, trauma, virus, bacteria (gram positive or gram negative), asystole preceding PICU admission, use of inotrope, anticonvulsant, seizures, raised intracranial pressure, encephalitis, disseminated intravascular coagulopathy, transfusion of blood or blood products.

One outlier with acute disseminated encephalomyelitis and respiratory failure stayed at the PICU for 1038 days. He was excluded from the calculation.

\section{Discussion}

PICU admissions are generally associated with a low but definite risk of mortality [2,14-16]. There has been no significant change in trend and the low mortality rates are similar to those reported from the United Kingdom $(5.1 \%)$ and Europe $(5.8 \%)[2,4,14,16]$. Infants account for one-fourth and males account for $61 \%$ of PICU deaths. The median PICU stay between admission and death was generally brief (median stay 3.5 days) and compatible with durations reported in other series $[2,4,17]$. Gender disparity in childhood morbidity has been demonstrated in a large study involving 92,332 pediatric admissions [18]. There is no relevant demographic difference between the two genders in terms of mortality in this series.

Patients who die in the PICU generally have multiorgan system involvement as evinced by the high rates of mechanical ventilation, inotrope and broad antibiotic usage. Septicemia is associated with a number of PICU deaths and may involve bacterial, fungal and viral pathogens [19]. Nevertheless, some patients have received antibiotics prior to PICU admissions, rendering the blood

Table 4 Length-of-survival analysis by multivariate Cox-Regression forward stepwise test

\begin{tabular}{lllll}
\hline & B (SE) & Exp (B) & $\mathbf{9 5 \% ~ C l ~ f o r ~} \operatorname{Exp}(\mathbf{B})$ & $\boldsymbol{p}$ \\
\hline NRCPR & 0.61 & 1.84 & $1.18-2.86$ & 0.007 \\
Fungal isolates & -0.79 & 0.45 & $0.26-0.80$ & 0.007 \\
Bacteremia & 0.59 & 1.81 & $1.08-3.03$ & 0.024 \\
Mechanical ventilation & -1.01 & 0.36 & $0.17-0.80$ & 0.011 \\
\hline
\end{tabular}

Exp (B): Hazard ratio for death. 
Table 5 Multivariate Cox-Regression forward stepwise test showed none of the following factors were associated with decision for NRCPR or DNAR

\begin{tabular}{|c|c|c|}
\hline Variables* & Score & $\mathbf{p}$ \\
\hline Number of prior PICU admissions & 0.912 & 0.34 \\
\hline Sex & 0.001 & 0.98 \\
\hline Age & 0.305 & 0.58 \\
\hline Oncologic diagnosis & 0.43 & 0.51 \\
\hline Trauma & 0.51 & 0.47 \\
\hline Virus & 0.001 & 0.97 \\
\hline Fungus & 1.80 & 0.18 \\
\hline Bacteria in $\mathrm{BC}$ & 0.62 & 0.43 \\
\hline Gram positive bacteria in $B C$ & 1.14 & 0.29 \\
\hline Gram negative bacteria in $B C$ & 0.021 & 0.89 \\
\hline Any bacteria & 0.13 & 0.72 \\
\hline Any Gram positive bacteria & 1.35 & 0.25 \\
\hline Any Gram negative bacteria & 0.23 & 0.63 \\
\hline Any infection & 0.22 & 0.64 \\
\hline Mechanical ventilation & 1.33 & 0.25 \\
\hline Asystole preceding PICU & 0.88 & 0.35 \\
\hline Inotrope usage prior to CPR & 0.12 & 0.73 \\
\hline Anticonvulsant usage & 1.19 & 0.28 \\
\hline Seizure/encephalitis/raised intracranial pressure & 1.87 & 0.17 \\
\hline Transfusion & 2.01 & 0.16 \\
\hline Platelets or fresh frozen plasma transfusion & 1.83 & 0.18 \\
\hline Disseminated intravascular coagulopathy & 0.014 & 0.91 \\
\hline Systemic antibiotics & 0.007 & 0.94 \\
\hline Systemic corticosteroid & 0.074 & 0.79 \\
\hline PIM 2 & 0.78 & 0.38 \\
\hline
\end{tabular}

*Residual Chi-Squares are not computed because of redundancies.

cultures negative despite the clinical presentation being consistent with sepsis. Importantly, adequate coverage for gram negative bacteria, staphylococci, streptococci, fungi and cytomegalovirus should be considered especially in immuno-compromised patients and patients with hematologic/oncologic disorders [20,21]. Sensible initial antimicrobial coverage targeting potential pathogens must be promptly instituted.

With or without infections, hematologic/oncologic diagnoses were the leading category among the non-trauma PICU deaths, and majority had hematological malignancies [20]. Our findings were consistent with data described at other PICUs [9,10,22-24]. Trauma is a common and important cause of pediatric mortality $[2,7,11,12,25]$. The mechanisms of injury vary widely [8]. Falls account for nearly half of the deaths. Drowning or submersion injury represents another small but potentially preventable cause of mortality $[6,7,11]$. In this series, no infants died from trauma or non-accidental injury. Interestingly, there has been no pediatric gunshot death in Hong Kong due to strict local gun-control regulations. This is very different from countries where gunshots contribute to a significant part of pediatric death $[7,8,11,26]$ PICU deaths associated with trauma showed a high percentage of male due possibly to curiosity and accident-prone behavior in boys [27]. More than half of the trauma deaths in our study were due to head injuries and subsequent brain-death. In addition, PIM2 score of the trauma associated deaths was significantly higher than that of the non-trauma related deaths. This might be due to the fact that significantly more patients in the trauma group presented in asystole before PICU admission, which contributes to "high risk diagnosis" in calculation of the PIM 2 score [13].

Deaths in PICU patients with metabolic diseases were relatively rare and often difficult to confirm. A similar study in the UK showed that $4.4 \%$ of the PICU non-survivors were diagnosed of metabolic diseases [2]. Chromosomal abnormalities and neurodegenerative diseases are also uncommon in our group of patients.

The decision and implementation of an end-of-life plan is a multi-faceted process that takes time and patience to eventuate [14,16,28]. Resuscitation was not attempted at the time of their deaths in nearly half of the patients in honor of caregivers' wishes. Parents often make timely DNAR decision when futility of treatment becomes evident. As a result, DNAR patients may paradoxically appear to take a lot longer to inevitably die. Despite prolonged non-survival, caregivers can be assured that DNAR patients generally received the same level of PICU supports including hemodynamic monitoring, mechanical ventilation, inotrope and antimicrobial usage as NRCPR patients during their PICU course.

There were only two PICU cases of organ donation following brain deaths, and both patients were from non-Chinese families (US and Japanese). Organ donations are rare in Chinese families possibly related to ethnic beliefs [29]. These patients typically did not have active infection.

Limitation of this study was the relative small sample size and heterogeneity despite the long study period of over 10 years. This may be due to the advanced medical care and relative small pediatric population in the aging Hong Kong demographics. In addition, difficulties were encountered in retrieving some of the patients' records due to the retrospective nature of the study. Nevertheless, data from CMS and autopsy reports are highly reliable. Lastly, our study only investigates PICU deaths and excludes children who die prior to hospitalization or PICU admission.

\section{Conclusion}

In conclusion, PICU admissions were associated with a $5.8 \%$ mortality rate. Death in the PICU is a heterogeneous 
event that involves infants and children. Resuscitation was not attempted at the time of their deaths in nearly half of the patients in honor of parents' wishes. Parents often make DNAR decision when medical futility becomes evident. Despite prolonged non-survival, DNAR patients generally received the same level of PICU supports as NRCPR patients during their PICU course.

\section{Competing interests}

The authors declare that they have no competing interests.

\section{Authors' contributions}

$\mathrm{KLHH}$ is the principal author. TCWP is the scientific staff who performed detailed statistical analysis. WW, KLC, HMC, TFL, CKL wereinvolved in the care of these patients and contributed to the design and drafting of this manuscript. KFT is the pathologist involved in post-mortum analysis of some of these patients. KKL, HWM, KWT, WKW, HFW wereinvolved in the data collection and analysis. AKCL was involved in the interpretation of the data and revision of the manuscript critically for important intellectual content. All authors read and approved the final manuscript.

\section{Author details}

${ }^{1}$ Department of Pediatrics, The Chinese University of Hong Kong, 6/F, Clinical Science Building, Prince of Wales Hospital, Shatin, Hong Kong, SAR, China ${ }^{2}$ Faculty of Medicine, The Chinese University of Hong Kong, Shatin, New Territories, Hong Kong. ${ }^{3}$ Department of Anatomical and Cellular Pathology, The Chinese University of Hong Kong, Shatin, New Territories, Hong Kong. ${ }^{4}$ Department of Pediatrics, The University of Calgary, 2500 University Dr NW, Calgary AB T2N 1N4, Canada.

Received: 4 February 2013 Accepted: 6 November 2013

Published: 17 November 2013

\section{References}

1. Balk RA: Pathogenesis and management of multiple organ dysfunction or failure in severe sepsis and septic shock. Crit Care Clin 2000, 16(2):337-352. vii.

2. Sands R, Manning JC, Vyas H, Rashid A: Characteristics of deaths in paediatric intensive care: a 10-year study. Nurs Crit Care 2009, 14(5):235-240.

3. Gruenberg DA, Shelton W, Rose SL, Rutter AE, Socaris S, McGee G: Factors influencing length of stay in the intensive care unit. Am J Crit Care 2006, 15(5):502-509.

4. Strand K, Walther SM, Reinikainen M, Ala-Kokko T, Nolin T, Martner J, et al: Variations in the length of stay of intensive care unit nonsurvivors in three scandinavian countries. Crit Care 2010, 14(5):R175.

5. Hon KL, Hung E, Tang J, Chow CM, Leung TF, Cheung KL, et al: Premorbid factors and outcome associated with respiratory virus infections in a pediatric intensive care unit. Pediatr Pulmonol 2008, 43(3):275-280.

6. Hon KL, Leung TF, Chan SY, Cheung KL, Ng PC: Indoor versus outdoor childhood submersion injury in a densely populated city. Acta Paediatr 2008, 97(9):1261-1264.

7. Hon KL, Leung TF, Cheung KL, Nip SY, Ng J, Fok TF, et al: Severe childhood injuries and poisoning in a densely populated city: where do they occur and what type? J Crit Care 2010, 25(1):175-12.

8. Rimsza ME, Schackner RA, Bowen KA, Marshall W: Can child deaths be prevented? the arizona child fatality review program experience. Pediatrics 2002, 110(1 Pt 1):e11.

9. Meyer S, Gottschling S, Gortner L: The prognosis of children with cancer in the PICU. J Pediatr Hematol Oncol 2009, 31(12):990-991.

10. Dursun O, Hazar V, Karasu GT, Uygun V, Tosun O, Yesilipek A: Prognostic factors in pediatric cancer patients admitted to the pediatric intensive care unit. J Pediatr Hematol Oncol 2009, 31(7):481-484.

11. Hon KL, Leung AK: Childhood accidents: injuries and poisoning. Adv Pediatr 2010, 57(1):33-62.

12. Hon KL, Chan J, Cheung KL: Head injuries after short falls: different outcomes despite similar causes. Hong Kong Med J 2010, 16(6):497-498.

13. Slater A, Shann F, Pearson G: Paediatric index of mortality P. PIM2: a revised version of the paediatric index of mortality. Intens Care Med 2003, 29(2):278-285
14. Devictor DJ, Nguyen DT: Forgoing life-sustaining treatments in children: a comparison between Northern and Southern European pediatric intensive care units. Pediatr Crit Care Med 2004, 5(3):211-215.

15. El Halal MG, Barbieri E, Filho RM, Trotta EA, Carvalho PR: Admission source and mortality in a pediatric intensive care unit. Indian J Crit Care Med 2012, 16(2):81-86.

16. Devictor DJ, Latour JM: Forgoing life support: how the decision is made in European pediatric intensive care units. Intensive Care Med 2011, 37(11):1881-1887.

17. Vernon DD, Dean JM, Timmons OD, Banner W Jr, Allen-Webb EM: Modes of death in the pediatric intensive care unit: withdrawal and limitation of supportive care. Crit Care Med 1993, 21(11):1798-1802.

18. Hon KL, Nelson EA: Gender disparity in paediatric hospital admissions. Ann Acad Med Singapore 2006, 35(12):882-888

19. Gray J, Gossain S, Morris K: Three-year survey of bacteremia and fungemia in a pediatric intensive care unit. Pediatr Infect Dis J 2001, 20(4):416-421.

20. Cugno C, Cesaro S: Epidemiology, risk factors and therapy of candidemia in pediatric hematological patients. Pediatr Rep 2012, 4(1):e9.

21. Brissaud O, Guichoux J, Harambat J, Tandonnet O, Zaoutis T: Invasive fungal disease in PICU: epidemiology and risk factors. Ann Intensive Care 2012, 22(2(1)):6.

22. Haase R, Lieser U, Kramm C, Stiefel M, Vilser C, Bernig T, et al: Management of oncology patients admitted to the paediatric intensive care unit of a general children's hospital - a single center analysis. Klin Padiatr 2011, 223(3):142-146.

23. Akhtar N, Fadoo Z, Panju S, Haque A: Outcome and prognostic factors seen in pediatric oncology patients admitted in PICU of a developing country. Indian J Pediatr 2011, 78(8):969-972.

24. Davidoff AM: Pediatric oncology. Semin Pediatr Surg 2010, 19(3):225-233

25. Hon KL: Dying with parents: an extreme form of child abuse. World J Pediatr 2011, 7(3):266-268.

26. Hon KL: No guns at children: not even a toy one! Indian J Pediatr 2011 78(12):1556-1557.

27. Vital E, Oliveira R, Do Ceu MM, De Matos MG: Injuries and risk-taking behaviours in Portuguese adolescents: highlights from the health behaviour in school-aged children survey. J Safety Res 2011, 42(5):327-331.

28. Carvalho PR, Rocha TS, Santo AE, Lago P: Modes of death in the PICU of a tertiary care hospital. Rev Assoc Med Bras 2001, 47(4):325-331.

29. Molzahn AE, Starzomski R, McDonald M, O'Loughlin C: Chinese Canadian beliefs toward organ donation. Qual Health Res 2005, 15(1):82-98.

doi:10.1186/1471-2253-13-43

Cite this article as: Hon et al:: Prolonged non-survival in PICU: does a do-not-attempt-resuscitation order matter. BMC Anesthesiology 2013 13:43.

\section{Submit your next manuscript to BioMed Central and take full advantage of:}

- Convenient online submission

- Thorough peer review

- No space constraints or color figure charges

- Immediate publication on acceptance

- Inclusion in PubMed, CAS, Scopus and Google Scholar

- Research which is freely available for redistribution 